\title{
Syntheses and antimicrobial activities of amide derivatives of 4-[(2-isopropyl-5-methylcyclohexyl)oxo]-4-oxobutanoic acid
}

\author{
Auj e Sana ${ }^{1}$, Sher Wali Khan ${ }^{1}$, Javid H. Zaidi ${ }^{{ }^{*}}$, Nida Ambreen ${ }^{2}$, Khalid Mohammed Khan ${ }^{2^{*}}$, \\ Shahnaz Perveen ${ }^{3}$ \\ ${ }^{1}$ Department of Chemistry, Quaid-i-Azam University, Islamabad, Pakistan; ${ }^{*}$ Corresponding Author: javid zaidi@yahoo.com \\ ${ }^{2}$ H. E. J. Research Institute of Chemistry, International Center for Chemical and Biological Sciences, University of Karachi, Karachi, \\ Pakistan; ${ }^{*}$ Corresponding Author: hassaan2@super.net.pk \\ ${ }^{3}$ PCSIR Laboratories Complex, Karachi, Shahrah-e-Dr, Salimuzzaman Siddiqui, Karachi, Pakistan.
}

Received 1 August 2011; revised 3 September 2011; accepted 11 September 2011.

\begin{abstract}
Chiral 4-[(2-isopropyl-5-methylcyclohexyl)oxo]-4oxobutanoic acid reacts with substituted anilines to produce amides 1-6 in high yields. Resulted amides 1-6 were investigated for their antifungal and antibacterial activities. Compounds $2(96.5 \%)$ against Aspergillus fumigatus and $6(93.7 \%)$ against Helminthosporium sativum demonstrated excellent activities. However, compounds 3 (37.6\%) against Bacillus subtilis, 4 (33.2\%) against $P$ seudomonas aurignosa, 5 against Klebsiella pneumonia demonstrated excellent growth inhibition potential.
\end{abstract}

Keywords: Chiral 4-[(2-lsopropyl-5-methylcyclohexyl)oxo]-4-oxobutanoic Acid; Substituted Anilines;

Amides; Antimicrobial Activity

\section{INTRODUCTION}

The growing incidence of bacterial resistance to existing antibiotics poses a serious medical problem in treating pathogenic infections $[1,2]$. Hence, there is an urgent need for molecules which are more potent and less sensitive to developing resistance properties than currently in use clinical antibiotics [3].

Succinic acid is predicted to be one of the future platform chemicals that can be derived from renewable resources. The amide bond is the most important linkage in organic chemistry and possesses the key functional group in peptides, polymers, in many natural products and pharmaceuticals $[4,5]$. Amides are synthesized by coupling of carboxylic acids and amines by the use of either a coupling reagent [6] or by prior conversion of the carboxylic acid into a derivative [7]. Alternative procedures involve the Staudinger ligation [8], aminocarbonylation of aryl halides [9] and oxidative amidation of aldehydes [10]. The preparation of amides and their physical and chemical properties are extensively documented $[11,12]$. The formation of amides on solid support usually involves reactions of amines either with acid halides $[13,14]$ or anhydrides [15] in the presence of base, or with acids in the presence of coupling agents [16-18] such as 1-hydroxybenzotriazole (HOBt), 7-aza-1-hydroxybenzotriazole (HOAt), or their ammonium or phosphonium salts. The formation of amides and analogues such as ureas, urethanes and thioureas, on solid support had also been reviewed $[19,20]$.

Herein, we report improvements in biological propeties of chiral 4-[(2-isopropyl-5-methylcyclohexyl)oxo]4-oxobutanoic acid amide derivatives. Chiral 4-[(2-isopropyl-5-methylcyclohexyl)oxo]-4-oxobutanoic acid (mono ester of succinic acid) was used as starting material which was derived from succinic anhydride by reacting with $S$ $(+)$ menthol. In view of various biological properties are associated with amides therefore, it seemed interesting to synthesize chiral amide derivatives of succinic acid. They were prepared by reaction of different aromatic amines with chiral 4-[(2-isopropyl-5-methylcyclohexyl)oxo]-4oxobutanoic acid to afford the corresponding amides. Six chiral amides derivatives 1-6 were synthesized and their structure had been characterized by the UV, IR, ${ }^{1} \mathrm{H}-\mathrm{NMR},{ }^{13} \mathrm{C}-\mathrm{NMR}$ and mass spectroscopic analysis. All six derivatives were screened for their antimicrobial activities.

\section{RESULTS AND DISCUSSION}

\subsection{Chemistry}

Owing to the importance of amides in organic synthe- 
sis, we planned to synthesize some new biologically active amides. In our research work we have selected an inexpensive and commercially available starting material succinic anhydride. Six amides were synthesized by the outlined route as shown in Scheme 1.

Chiral menthol ester was synthesized by using our previously reported method [21]. Amide functionality was being introduced by reaction of mono ester with different substituted anilines in $\mathrm{CHCl}_{3}$ in the presence of $N, N$-dicyclohexylcarbodiimide (DCC). All the synthesized compounds were purified by column chromatography and characterized by using spectroscopic techniques like IR, ${ }^{1} \mathrm{H}-\mathrm{NMR}$ and ${ }^{13} \mathrm{C}-\mathrm{NMR}$ spectroscopy. Compound 1-6 also gave satisfactory elemental analyses.

IR spectra of all six amides displayed $\mathrm{C}=\mathrm{O}$ (amide) absorption at $1701-1728 \mathrm{~cm}^{-1}$ beside $\mathrm{C}=\mathrm{O}$ (ester) absorption at $1649-1696 \mathrm{~cm}^{-1}$. In ${ }^{13} \mathrm{C}-\mathrm{NMR}$ spectra $\mathrm{C}=\mathrm{O}$ resonated at $169 \mathrm{ppm}$ for ester and at $172 \mathrm{ppm}$ for amide. In each case, it indicating that an amide linkage has been established. In ${ }^{1} \mathrm{H}-\mathrm{NMR}$ protons of $-\mathrm{CH}_{2}$ groups of succinic acid moiety resonated at $2.65 \mathrm{ppm}$ and $2.73 \mathrm{ppm}$ as triplet integrating for two protons each rather than doublet of triplet indicating the formation of corresponding amide.

In case of compounds $\mathbf{3}$ and $\mathbf{6}$ aromatic protons resonated at 6.84 - $7.44 \mathrm{ppm}$ and 7.11 - $7.41 \mathrm{ppm}$, respecttively, as two doublets indicating the para substitution. Complete ${ }^{1} \mathrm{H}$-NMR and ${ }^{13} \mathrm{C}$-NMR data is given in experimental section. Elemental analysis values are in acceptable range provide an additional evidence to establish structures of synthesized compounds.

\subsection{Bioassay}

All six synthetic amides 1-6 were tested to study their antimicrobial activities against three selected fungal strains and four bacterial strains. Following methods were used to check these activities. DMSO was used as control solvent, terbinafin, and penicillium were used as standard drugs for antifungal and antibacterial activities, respectively.

\subsubsection{Antifungal Activity}

All six synthetic amides 1-6 showed varying degree of growth inhibition against all tested fungal strains and results are collected in Table $\mathbf{1 .}$

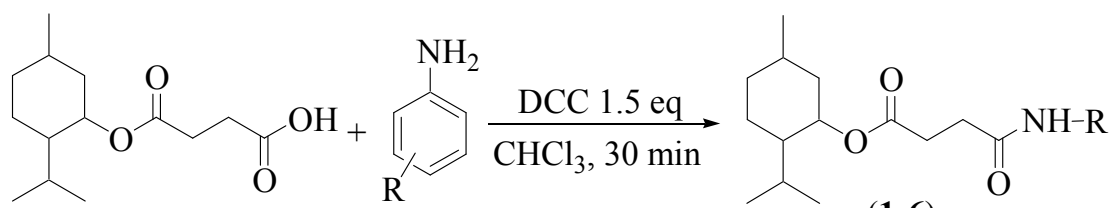

(1-6)

\begin{tabular}{|c|c|c|c|}
\hline S. No & Compound No. & $R$ & Isolated Yield (\%) \\
\hline 1 & $\mathbf{1}$ & & \\
\hline 2 & $\mathbf{2}$ & & \\
\hline 3 & $\mathbf{3}$ & \\
\hline 4 & $\mathbf{4}$ & \\
\hline 5 & $\mathbf{5}$ & \\
\hline 6 & $\mathbf{6}$ & \\
\hline
\end{tabular}

Scheme 1. Synthesis of amides from chiral 4-[(2-isopropyl-5-methylcyclohexyl)oxo]-4-oxobutanoic acid. 
Compound 2 exhibited an excellent percentage growth inhibition (96.5\%) against Aspergillus fumigatus better than standard terbinafin $(96.3 \%)$ in the same conditions. Compound 6 also demonstrated an excellent percentage growth inhibition (93.7) against Helminthosporium sativum strain also superior to terbinafin (92.7\%). Compounds 1 (86.9\%), 3 (85.7\%), 4 (79.0\%) exhibited appreciable percentage growth inhibitions against Aspergillus fumigatus, Fusarium moniliforme, and Helminthosporium sativum, respectively. Compounds 5 (70.7\%) and $6(73.7 \%)$ showed considerable percentage growth inhibitions against Aspergillus fumigatus. Compounds 2 (68.8\%), 4 (66.2\%), 5 (68.8\%), and $6(63.6 \%)$ were found to be significantly active against Fusarium moniliforme fungal strain. However, compounds 1 (55\%), 2 (32\%), 3 (39\%), and 5 (43\%) showed moderate to weak activities against Helminthosporium sativum.

\subsubsection{Antibacterial Activity}

All six synthetic amides 1-6 showed varying degree of percentage inhibition against all four selected bacterial strains and results are summarized in Table 2.

Table 1. Antifungal activities of the synthesized amides 1-6.

\begin{tabular}{|c|c|c|c|c|c|c|}
\hline \multirow{2}{*}{$\begin{array}{l}\text { Treatments } \\
\begin{array}{l}\text { Compounds } \\
(15 \mathrm{mg} / \mathrm{mL})\end{array}\end{array}$} & \multicolumn{2}{|c|}{ Aspergillus fumigatus } & \multicolumn{2}{|c|}{ Fusarium moniliforme } & \multicolumn{2}{|c|}{ Helminthosporium sativum } \\
\hline & $\begin{array}{l}\text { Mycelial growth } \\
(\mathrm{cm})\end{array}$ & $\begin{array}{c}\text { Growth inhibition } \\
(\%)\end{array}$ & $\begin{array}{l}\text { Mycelial growth } \\
(\mathrm{cm})\end{array}$ & $\begin{array}{c}\text { Growth inhibition } \\
(\%)\end{array}$ & $\begin{array}{l}\text { Mycelial growth } \\
(\mathrm{cm})\end{array}$ & $\begin{array}{c}\text { Growth inhibition } \\
(\%)\end{array}$ \\
\hline Control & 9.9 & $\mathbf{0}$ & 7.7 & $\mathbf{0}$ & 10 & $\mathbf{0}$ \\
\hline 1 & 1.3 & 86.9 & 5.5 & 28.6 & 4.5 & $\mathbf{5 5 . 0}$ \\
\hline 2 & 5.4 & 96.5 & 2.4 & 68.8 & 1.8 & 32.0 \\
\hline 3 & 6.1 & 38.4 & 1.1 & 85.7 & 6.1 & 39.0 \\
\hline 4 & 5.1 & 51.5 & 2.6 & 66.2 & 2.1 & 79.0 \\
\hline 5 & 2.9 & 70.7 & 2.4 & 68.8 & 5.7 & 43.0 \\
\hline 6 & 2.6 & 73.7 & 2.8 & 63.6 & 6.8 & 93.7 \\
\hline Terbinafin (Std.) & 0.37 & 96.3 & 0.35 & 95.5 & 0.73 & 92.7 \\
\hline
\end{tabular}

Table 2. Antibacterial activities of the synthetic amides 1-6.

\begin{tabular}{|c|c|c|c|c|c|}
\hline \multirow{2}{*}{ S. No. } & \multirow{2}{*}{ Compounds } & \multirow{2}{*}{$\begin{array}{c}\text { Staphylococcus aureus } \\
\% \text { Inhibition }\end{array}$} & \multirow{2}{*}{$\begin{array}{c}\text { Pseudomonas aurignosa } \\
\% \text { Inhibition }\end{array}$} & \multirow{2}{*}{$\begin{array}{c}\text { Bacillus subtilis } \\
\% \text { Inhibition }\end{array}$} & \multirow{2}{*}{$\begin{array}{c}\text { Klebsiella pneumonia } \\
\% \text { Inhibition }\end{array}$} \\
\hline & & & & & \\
\hline 1 & 1 & 29.6 & 10.6 & 15.3 & 20.6 \\
\hline 2 & 2 & 20 & 10.5 & 18.3 & 36 \\
\hline 3 & 3 & 16.3 & 12.6 & 37.6 & 19 \\
\hline 4 & 4 & 15.3 & 33.2 & 16.3 & 21.3 \\
\hline 5 & 5 & 28.3 & 14.6 & 24 & 39.6 \\
\hline 6 & 6 & 18.3 & 11 & 22 & 19 \\
\hline \multicolumn{2}{|c|}{ Penicillium (Std.) } & 30.3 & 32.3 & 34.3 & 38.6 \\
\hline
\end{tabular}

Concentration $=15 \mathrm{mg} / \mathrm{mL}$. 
Compound 3 exhibited an excellent percentage inhibition (37.6\%) against Bacillus subtilis superior to standard penicillium (34.3\%) in the same conditions. Compound 4 also showed an excellent percentage inhibition (33.2\%) against Pseudomonas aurignosa better than standard penicillium (32.3\%). Compounds 5 demonstrated a strong percentage inhibition (37.6\%) against Klebsiella pneumonia also superior to penicillium (38.6\%). Compound 1 found to have a comparable inhibition against Staphylococcus aureus (29.6\%) to standard penicillium (30.3\%). Compound 2 (36\%) demonstrated a appreciable growth inhibition against Klebsiella pneumonia. However, compounds 5 also exhibited a good growth inhibition against Staphylococcus aureus with a percentage inhibition of 28.3. Compounds 2 (20\%), 3 (16.3\%), 4 (15.3\%), and 6 $(18.3 \%)$ showed moderate to weak activities against Staphylococcus aureus. Compounds $1(10.6 \%), 2$ (10.5\%), $3(12.6 \%), 5(14.6 \%)$, and $\mathbf{6}(11 \%)$ exhibited moderate to weak growth inhibitions against Pseudomonas aurignosa. Compounds 1 (15.3\%), 2 (18.3\%), 4 (16.3\%), 5 (24\%) and 6 (22\%) were found to have moderate to weak activities against Bacillus subtilis. In addition, compound 1 (20.6\%), 3 (19\%), 4 (21.3), and 6 (19\%) showed moderate to weak activities against Klebsiella pneumonia.

\section{CONCLUSIONS}

Compounds 2 (96.5\%) against Aspergillus fumigatus and 6 (93.7\%) against Helminthosporium sativum demonstrated excellent activities and may be served as lead compounds for further research on these molecules as useful antifungal agents. However, compounds 3 (37.6\%) against Bacillus subtilis, 4 (33.2\%) against Pseudomonas aurignosa, $\mathbf{5}$ against Klebsiella pneumonia demonstrated excellent growth inhibition potential therefore may be served as lead compounds for further research on these compounds in search of better antibacterial agents.

\section{EXPERIMENTAL}

All reactions were carried out in anhydrous conditions and under static pressure of nitrogen gas using rubber septa and three way stopcock. Solvent like ether was dried and distilled over sodium and benzophenone. Chloroform was dried by refluxing with phosphorus pentoxide and methanol dried with magnesium turnings and iodine crystals. Amines were dried by refluxing over potassium hydroxide. All the reactions were monitored through thin layer chromatography using pre-coated silica gel glass plates ( $0.25 \mathrm{~mm}, \mathrm{HF}-254$, E. Merck). Methanol and chloroform mixture were used as eluent. Chromatograms were visualized using ultraviolet light at $\lambda_{\max }$ 254 or $365 \mathrm{~nm}$. Column chromatography was performed on silica gel (0.063 - $0.200 \mathrm{~mm}$ E. Merck).
FTIR spectra were recorded on Schimadzu Fourier Transform Infrared Spectrophotometer Model 270. Solid samples were taken in $\mathrm{KBr}$ pellets and oils were used in $\mathrm{NaCl}$ cell for recording their spectra. ${ }^{1} \mathrm{H}-\mathrm{NMR}$ spectra were recorded on NMR Bruker apparatus at $300 \mathrm{MHz}$ in $\mathrm{CDCl}_{3} .{ }^{13} \mathrm{C}$-NMR spectra were recorded on NMR Bruker apparatus at $75 \mathrm{MHz}$. Tetramethylsilane (TMS) was used as internal reference. Chemical shifts are given in $\delta$ (ppm) and abbreviations s, d, and t have been used for singlet, doublet and triplet, respectively. The optical rotations of the compounds were measured on ATAGO, AP-100 automatic polarimeter.

\subsection{General Procedure}

In $50 \mathrm{~mL}$ conical flask a mixture of 4-(2-isopropyl-5methylcyclohexyloxy)-4-oxobutan-oic acid and (0.004 mol, $0.418 \mathrm{~g})$ and substituted aniline (1 g $0.004 \mathrm{~mol})$ were taken and added DCC $(0.005 \mathrm{~mol}, 1 \mathrm{~g})$ dissolved in $250 \mathrm{~mL}$ of dry $\mathrm{CHCl}_{3}$ and stirred for half an hour. White crystals of urea were filtered off and chloroform was removed under reduced pressure. Residue was dissolved in ethyl acetate, filtered and solvent was evaporated under reduced pressure. The resulting product was purified by column chromatography. The purity of the product was checked with TLC in 10\% methanol in chloroform.

\subsection{1. (1r,2r,5s)-2-Isopropyl-5-methylcy-clohexyl- 4-(2-methoxyphenylamino)-4-oxo-butanoate} (1)

Yield $88 \%,[\alpha]^{23}=-5.94$ (Chloroform, Conc. $=10 \mathrm{mg} /$ 20 mL). FTIR: 3337 (NH Str), 2928 (CH Ar), 2855 (CH aliphatic), 1725 (CO ester), $1696 \mathrm{~cm}^{-1}$ (CO amide), ${ }^{1} \mathrm{H}-\mathrm{NMR}\left(300 \mathrm{MHz}, \mathrm{CDCl}_{3}\right): \delta 0.76(\mathrm{~d}, 3 \mathrm{H}, J=9 \mathrm{~Hz})$, $0.91\left(\mathrm{dt}, 6 \mathrm{H}, J_{3}=6 \mathrm{~Hz}, J_{5}=3 \mathrm{~Hz}\right), 1.48(\mathrm{~m}, 6 \mathrm{H}), 1.69(\mathrm{~m}$, $1 \mathrm{H}), 1.88(\mathrm{~m}, 1 \mathrm{H}), 1.99(\mathrm{~m}, 1 \mathrm{H}), 2.71(\mathrm{t}, 2 \mathrm{H}, J=6 \mathrm{~Hz})$, $2.31(\mathrm{t}, 2 \mathrm{H}, J=6 \mathrm{~Hz}),$,3.89 (s, $3 \mathrm{H}), 4.70\left(\mathrm{dt}, 1 \mathrm{H}, J_{3}=9\right.$ $\left.\mathrm{Hz}, J_{5}=3 \mathrm{~Hz}\right) 6.86-7.07(\mathrm{~m}, 5 \mathrm{H}),{ }^{13} \mathrm{C}-\mathrm{NMR}(75 \mathrm{MHz}$, $\left.\mathrm{CDCl}_{3}\right): 173.2,172.4,119.7$ - 154.1, 74.8, 55.6, 46.9, 40.8, 34.2, 32.6, 29.9, 28.9, 26.2, 22.7, 22.0, 16.3; Anal. Calcd for $\mathrm{C}_{21} \mathrm{H}_{31} \mathrm{NO}_{4}(361.48) \mathrm{C}, 69.78 ; \mathrm{H}, 8.64 ; \mathrm{N}, 3.87$; O, 17.70; Found: C, 69.93; H, 8.71; N, 3.75; O, 17.61.

\subsection{2. (1R,2R,5S)-2-Isopropyl-5-methylcy-clohexyl- 4-(3-methoxyphenylamino)-4-oxo-buta noate (2)}

Yield $81 \%,[\alpha]^{23}{ }_{\mathrm{D}}=+0.16$ (Chloroform, Conc. $=10 \mathrm{mg}$ / 20 mL). FTIR: 3324 (NH Str), 2927 (CH Ar), 2854 (CH aliphatic), 1701 (CO ester), $1654 \mathrm{~cm}^{-1}$ (CO amide), ${ }^{1} \mathrm{H}-\mathrm{NMR}\left(300 \mathrm{MHz}, \mathrm{CDCl}_{3}\right): \delta 0.76(\mathrm{~d}, 3 \mathrm{H}, J=9 \mathrm{~Hz})$, $0.91\left(\mathrm{dt}, 6 \mathrm{H}, J_{3}=6 \mathrm{~Hz}, J_{5}=3 \mathrm{~Hz}\right), 1.48(\mathrm{~m}, 6 \mathrm{H}), 1.69(\mathrm{~m}$, $1 \mathrm{H}), 1.88(\mathrm{~m}, 1 \mathrm{H}), 1.99(\mathrm{~m}, 1 \mathrm{H}), 2.66(\mathrm{t}, 2 \mathrm{H}, J=6 \mathrm{~Hz})$, $2.73(\mathrm{t}, 2 \mathrm{H}, J=6 \mathrm{~Hz}), 3.81(\mathrm{~s}, 3 \mathrm{H}), 4.69\left(\mathrm{dt}, 1 \mathrm{H}, J_{3}=\right.$ $\left.9 \mathrm{~Hz}, J_{5}=3 \mathrm{~Hz}\right), 7.12-7.57(\mathrm{~m}, 5 \mathrm{H}),{ }^{13} \mathrm{C}-\mathrm{NMR}(75 \mathrm{MHz}$, 
$\left.\mathrm{CDCl}_{3}\right): 173.2,172.4,115.6$ - 164.9, 74.8, 55.6, 46.9, 40.8, 34.2, 32.6, 29.9, 28.9, 26.2, 22.7, 22.0, 16.3; Anal. Calcd for $\mathrm{C}_{21} \mathrm{H}_{31} \mathrm{NO}_{4}(361.48) \mathrm{C}, 69.78 ; \mathrm{H}, 8.64 ; \mathrm{N}, 3.87$; O, 17.70; Found: C, 69.93; H, 8.71; N, 3.75; O, 17.61.

\subsection{3. (1R,2R,5S)-2-Isopropyl-5-methylcy-clohexyl- 4-(4-methoxyphenylamino)-4-oxo-butanoate} (3)

Grey crystalline solid. Yield $91 \%, \mathrm{~m} \cdot \mathrm{p}=141^{\circ} \mathrm{C},[\alpha]^{23}{ }_{\mathrm{D}}$ $=-1.69$ (Chloroform, Conc. $=10 \mathrm{mg} / 20 \mathrm{~mL}$ ). FTIR: 3301 (NH Str), 2919 (CH Ar), 2867 (CH aliphatic), 1724 $\mathrm{cm}$ (CO ester), $1655 \mathrm{~cm}^{-1}$ (CO amide), ${ }^{1} \mathrm{H}$ NMR (300 $\left.\mathrm{MHz}, \mathrm{CDCl}_{3}\right): \delta 0.76(\mathrm{~d}, 3 \mathrm{H}, J=9 \mathrm{~Hz}$ ), 0.91 (dt, $6 \mathrm{H}, J=$ $6 \mathrm{~Hz}, J=3 \mathrm{~Hz}), 1.48(\mathrm{~m}, 1 \mathrm{H}), 1.69(\mathrm{~m}, 1 \mathrm{H}), 1.88(\mathrm{~m}$, $1 \mathrm{H}), 1.99(\mathrm{~m}, 1 \mathrm{H}), 2.65(\mathrm{t}, 2 \mathrm{H}, J=6 \mathrm{~Hz}), 2.76(\mathrm{t}, 2 \mathrm{H}, J$ $=6 \mathrm{~Hz}), 3.80(\mathrm{~s}, 3 \mathrm{H}), 4.74(\mathrm{dt}, 1 \mathrm{H}, J=9 \mathrm{~Hz}, J=3 \mathrm{~Hz})$, 6.84 - $7.44(\mathrm{~m}, 5 \mathrm{H}),{ }^{13} \mathrm{C}-\mathrm{NMR}\left(75 \mathrm{MHz}, \mathrm{CDCl}_{3}\right): 173.2$, 172.4, 114.1 - 121.6, 74.8, 55.5 , 46.9, 40.8, 34.2, 32.6, 30.1, 28.9, 26.2, 22.7, 22.0, 16.3; Anal. Calcd for $\mathrm{C}_{21} \mathrm{H}_{31} \mathrm{NO}_{4}(361.48) \mathrm{C}, 69.78 ; \mathrm{H}, 8.64 ; \mathrm{N}, 3.87 ; \mathrm{O}, 17.70$; Found: $\mathrm{C}, 69.93 ; \mathrm{H}, 8.71 ; \mathrm{N}, 3.75 ; \mathrm{O}, 17.61$.

\subsection{4. (1R,2R,5S)-2-Isopropyl-5-methylcy-clohexyl- 4-oxo-4-(2-tolylamino)butanoate (4)}

Yield $87 \%,[\alpha]^{23}=-7.43$ (Chloroform, Conc. $=10$ $\mathrm{mg} / 20 \mathrm{~mL}$ ). FTIR: 3354 (NH Str), 2922 (CH Ar), 2852 (CH aliphatic), 1725 (CO ester), $1680 \mathrm{~cm}^{-1}$ (CO amide), ${ }^{1} \mathrm{H}-\mathrm{NMR}\left(300 \mathrm{MHz}, \mathrm{CDCl}_{3}\right): \delta 0.76(\mathrm{~d}, 3 \mathrm{H}, J=9 \mathrm{~Hz})$, 0.91 (dt, $6 \mathrm{H}, \mathrm{J} 3=6 \mathrm{~Hz}, J=3 \mathrm{~Hz}), 1.48(\mathrm{~m}, 6 \mathrm{H}), 1.59$ (s, $3 \mathrm{H}), 1.66$ (t, 2H, $J=6 \mathrm{~Hz}), 1.69(\mathrm{~m}, 1 \mathrm{H}), 1.78$ (t, 2H, $J$ $=6 \mathrm{~Hz}), 1.88(\mathrm{~m}, 1 \mathrm{H}), 1.99(\mathrm{~m}, 1 \mathrm{H}), 4.24(\mathrm{dt}, 1 \mathrm{H}, J=9$ $\mathrm{Hz}, J=3 \mathrm{~Hz}), 7.28-7.74(\mathrm{~m}, 5 \mathrm{H}),{ }^{13} \mathrm{C}-\mathrm{NMR}(75 \mathrm{MHz}$, $\left.\mathrm{CDCl}_{3}\right): 172.9,169.7,119.8-135.3,74.9,46.9,40.8$, 34.2, 32.4, 30.1, 28.9, 26.2, 22.7, 22.0, 20.9, 16.3; Anal. Calcd for $\mathrm{C}_{21} \mathrm{H}_{31} \mathrm{NO}_{3}(345.48) \mathrm{C}, 73.01 ; \mathrm{H}, 9.04 ; \mathrm{N}, 4.05$; O, 13.89; Found: C, 73.16; H, 9.25; N, 3.81; O, 13.78.

\subsection{5. (1R,2R,5S)-2-Isopropyl-5-methylcy-clohexyl- 4-oxo-4-(3-tolylamino)butanoate (5)}

Yield $87 \%,[\alpha]^{23}=-7.43$ (Chloroform, Conc $=10 \mathrm{mg} /$ 20 mL). FTIR: 3345 (NH Str), 2925 (CH Ar), 2855 (CH aliphatic), 1723 (CO ester), $1649 \mathrm{~cm}^{-1}$ (CO amide), ${ }^{1} \mathrm{H}-\mathrm{NMR}\left(300 \mathrm{MHz}, \mathrm{CDCl}_{3}\right): \delta 0.76(\mathrm{~d}, 3 \mathrm{H}, J=9 \mathrm{~Hz})$, $0.91(\mathrm{dt}, 6 \mathrm{H}, J=6 \mathrm{~Hz}, J=3 \mathrm{~Hz}), 1.48(\mathrm{~m}, 6 \mathrm{H}), 1.69(\mathrm{~m}$, $1 \mathrm{H}), 1.88(\mathrm{~m}, 1 \mathrm{H}), 1.97(\mathrm{~s}, 3 \mathrm{H}), 1.99(\mathrm{~m}, 1 \mathrm{H}), 2.19(\mathrm{t}$, $2 \mathrm{H}, J=6 \mathrm{~Hz}), 2.34$ (t, $2 \mathrm{H}, J=6 \mathrm{~Hz}), 4.24(\mathrm{dt}, 1 \mathrm{H}, J=9$ $\mathrm{Hz}, J=3 \mathrm{~Hz}), 7.26-7.73(\mathrm{~m}, 5 \mathrm{H}),{ }^{13} \mathrm{C}-\mathrm{NMR}(75 \mathrm{MHz}$, $\left.\mathrm{CDCl}_{3}\right): 172.9,169.7,119.8$ - 135.3, 74.9, 46.9, 40.8, $34.2,32.4,30.1,28.9,26.2,22.7,22.0,20.9,16.3$.

\subsection{6. (1R,2R,5S)-2-Isopropyl-5-methylcy-clohexyl- 4-oxo-4-(4-tolylamino)butanoate (6)}

White crystalline solid. Yield $96 \%$, m.p $=76^{\circ} \mathrm{C},[\alpha]^{23}{ }_{\mathrm{D}}$
$=-3.47$ (Chloroform, Conc $=10 \mathrm{mg} / 20 \mathrm{~mL}$ ). FTIR: 3328 (NH Str), 2919 (CH Ar), 2865 (CH aliphatic), 1728 (CO ester), $1660 \mathrm{~cm}^{-1}$ (CO amide), ${ }^{1} \mathrm{H}-\mathrm{NMR}(300 \mathrm{MHz}$, $\left.\mathrm{CDCl}_{3}\right): \delta 0.76(\mathrm{~d}, 3 \mathrm{H}, J=9 \mathrm{~Hz}), 0.91(\mathrm{dt}, 6 \mathrm{H}, J=6 \mathrm{~Hz}$, $J=3 \mathrm{~Hz}), 1.48(\mathrm{~m}, 6 \mathrm{H}), 1.69(\mathrm{~m}, 1 \mathrm{H}), 1.88(\mathrm{~m}, 1 \mathrm{H})$, $1.99(\mathrm{~m}, 1 \mathrm{H}), 2.32(\mathrm{~s}, 3 \mathrm{H}), 2.66(\mathrm{t}, 2 \mathrm{H}, J=6 \mathrm{~Hz}), 2.76(\mathrm{t}$, $2 \mathrm{H}, J=6 \mathrm{~Hz}), 4.74(\mathrm{dt}, 1 \mathrm{H}, J=9 \mathrm{~Hz}, J=3 \mathrm{~Hz}), 7.11$ $7.41(\mathrm{~m}, 5 \mathrm{H}),{ }^{13} \mathrm{C}-\mathrm{NMR}\left(75 \mathrm{MHz}, \mathrm{CDCl}_{3}\right): 172.9,169.7$, 119.8 - 135.3, 74.9, 46.9, 40.8, 34.2, 32.4, 30.1, 28.9, 26.2, 22.7, 22.0, 20.9, 16.3.

\subsection{Material and Method}

\subsubsection{Assay for Antifungal Activity}

The agar tube dilution method is used for determination of antifungal activity [22]. Fungal strains Aspergillus fumigatus, Fusarium moniliforme and Helminthosporium sativum were used in this study. Each fungal strain was maintained on Sabouraud's dextrose agar (Oxoid) medium at $4^{\circ} \mathrm{C}$.

The samples for antifungal assay were prepared from initial stock solution of $0.12 \mathrm{~g}$ of compound in $1 \mathrm{~mL}$ of dimethyl sulfoxide (DMSO). Culture media was prepared by dissolving $6.5 \mathrm{~g}$ of Sabouraud dextrose agar per $100 \mathrm{~mL}$ of distilled water $\mathrm{pH}$ was adjusted at 5.6. Test tubes were marked to the $10 \mathrm{~mL}$ mark. The Sabouraud's dextrose agar (Oxoid) dispensed as $10 \mathrm{~mL}$ volume into screw capped tubes or cotton plugged test tubes and were autoclaved at $121^{\circ} \mathrm{C}$ for 21 minutes. Tubes were allowed to cool to $50^{\circ} \mathrm{C}$ and Sabouraud's dextrose agar (Oxoid) agar was loaded with $67 \mu \mathrm{L}$ of compound pipette from the stock solution. This would give the final concentration of $200 \mu \mathrm{g} / \mathrm{mL}$ of the pure compound in media. Tubes were then allowed to solidify in slanting position at room temperature. Three slants of the compound sample were prepared for each fungus species. The tubes containing solidified media and sample compound were inoculated with $4 \mathrm{~mm}$ diameter piece of inoculums, taken from a seven days old culture of fungus. One sample of each compound was prepared, which was used for positive control. Slants without compound were used for negative control.

The test tubes were incubated at $28^{\circ} \mathrm{C}$ for 7 days. Cultures were examined twice weekly during the incubation. Reading was taken by measuring the linear length of fungus in slant by measuring growth $(\mathrm{mm})$ and growth inhibition was calculated with reference to negative control and all tests were carried out in triplicate.

\subsubsection{Assay for Antibacterial Activity}

Antibacterial activity of the methanolic solution of selected compounds was determined by agar well diffusion method [23]. Nutrient broth medium was prepared 
by dissolving $0.4 \mathrm{~g}$ of nutrient broth in $50 \mathrm{~mL}$ of distilled water. $\mathrm{pH}$ was adjusted at 7.0 and was sterilized by autoclaving. Nutrient agar medium was prepared by dissolving $2.3 \mathrm{~g}$ agar in $100 \mathrm{~mL}$ of distilled water; $\mathrm{pH}$ was adjusted at 7.0 and was autoclaved at $121^{\circ} \mathrm{C}$. Four strains of bacteria Staphylococcus aureus, Pseudomonas aurignosa, Bacillus subtilis and Klebsiella pneumonia were used in the study. The organisms were maintained on nutrient agar medium at $4^{\circ} \mathrm{C}$. Bacterial pallets obtained after centrifugation of $24 \mathrm{~h}$ old culture in nutrient broth of selected bacterial strains were mixed with physiological normal saline solution until a McFarland turbidity standard [106 colony forming unit (CFU) $\mathrm{mL}^{-1}$ ] was obtained. Then this inoculum was used for seeding the nutrient agar.

Nutrient agar medium was prepared by adding nutriaent agar $2.3 \mathrm{~g}$ in $100 \mathrm{~mL}$ of distilled water, $\mathrm{pH}$ was adjusted at 7.0, and was autoclaved. It was allowed to cool to $45^{\circ} \mathrm{C}$. Petri plates were prepared by pouring $75 \mathrm{~mL}$ of seeded nutrient agar and allowed to solidify. Four wells per plate were made with sterile cork borer $(5 \mathrm{~mm})$.

Using micropipette, $100 \mu \mathrm{L}$ of test solutions was poured in respective wells. These plates were incubated at $37^{\circ} \mathrm{C}$. After $24 \mathrm{~h}$ of incubation the diameter of the clear zones of inhibitions was measured by a ruler. Antibacterial activity of three dilutions of each compound was determined against three bacterial strains all tests were carried out in triplicate.

\section{ACKNOWLEDGEMENTS}

This work was financially supported by Higher Education Commission (HEC) Pakistan under "National Research Program for Universities."

\section{REFERENCES}

[1] James, J.K., Foster, J.R., Lentino, R.S. and Cathy D. (1986) Comparison of in vitro activity of quinolone antibiotics and vancomycin against gentamicin-And methicillin-Resistant Staphylococcus aureus by time-Kill kinetic studies. Antimicrobial Agents and Chemotherapy, 30, 823-827.

[2] Nwakaso, N., Umejiego, D., Gollapalli, L., Shaeling, A.J. and Lu, L.H. (2008) Targeting a prokaryotic protein in a eukaryotic pathogen: Identification of lead compounds against cryptosporidiosis. Chemistry \& Biology, 15, 7077. doi:10.1016/j.chembiol.2007.12.010

[3] Allemandi, D.A., Alovero, F.L. and Manzo, R.H. (1994) In vitro activity of new sulphanilyl fluoroquinolones against Staphylococcus aureus. Journal Antimicrobial Chemotherapy, 34, 261-264. doi:10.1093/jac/34.2.261

[4] Cupido, T., Tulla-Puche, J., Spengler, J. and Albericio, F. (2007) The synthesis of naturally occuring peptides and their analogs. Current Opinion in Drug Discovery and Development, 10, 768-783.
[5] Bode, J.W. (2006) Emerging methods in amide- and peptide-bond formation. Current Opinion in Drug Discovery and Development, 9, 765-775.

[6] Han, S.-Y. and Kim, Y.-A. (2004) Recent development of peptide coupling reagents in organic synthesis. Tetrahedron, 60, 2447-2467. doi:10.1016/j.tet.2004.01.020

[7] Montalbetti, C.A.G.N. and Falque, V. (2005) Amide bond formation and peptide coupling. Tetrahedron, 61, 1082710852. doi:10.1016/j.tet.2005.08.031

[8] Köhn, M. and Breinbauer, R. (2004) The staudinger ligation-A gift to chemical biology. Angewandte Chemie International Edition, 43, 3106-3116.

doi:10.1002/anie.200401744

[9] Martinelli, J.R., Clark, T.P., Watson, D.A., Munday, R.H., Buchwald, S.L. (2007) Palladium-catalyzed aminocarbonylation of aryl chlorides at atmospheric pressure: The dual role of sodium phenoxide. Angewandte Chemie International Edition, 46, 8460-8463. doi:10.1002/anie. 200702943

[10] Chang, J.W.W. and Chan, P.W.H. (2008) Highly efficient ruthenium (II) porphyrin catalyzed amidation of aldehydes. Angewandte Chemie International Edition, 47, 1138-1140. doi:10.1002/anie.200704695

[11] Bose, A.K., Manhas, M.S., Banik, B.K. and Srirajan, V. (2000) The amide linkage: Selected. Structural aspects in chemistry, biochemistry, and material science. In: Greenberg, A., Breneman, C.M. and Liebman J.F., Eds., WileyInterscience, New York, 157.

[12] Zabicky, J. (1970) The chemistry of amides interscience. London.

[13] Mohan, R., Chou, Y.-L. and Morrissey, M.M. (1996) Pictet-Spengler reaction on solid support: Synthesis of 1,2,3,4-tetrahydro- $\beta$-carboline libraries. Tetrahedron Letters, 37, 3963-3966. doi:10.1016/0040-4039(96)00773-3

[14] Moon, H.-S., Schore, N.E. and Kurth, M.J. (1994) A polymer-supported $\mathrm{C}_{2}$-symmetric chiral auxiliary: Preparation of non-racemic 3,5-disubstituted- $\gamma$-buty-rolactones. Tetrahedron Letters, 35, 8915-8918. doi:10.1016/0040-4039(94)88388-2

[15] Ceccato, M.-L., Chenu, J., Méry, J., Follet, M. and Calas, B. (1990) Glycolamidic ester handle used with poly-sty-

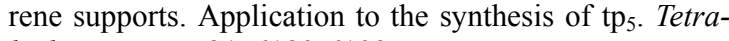
hedron Letters, 31, 6189-6192. doi:10.1016/S0040-4039(00)97021-7

[16] Li, P. and Xu, J.-C. (2000) HOBt and HOAt-derived immonium salts: New and highly efficient coupling reagents for peptide synthesis. Tetrahedron Letters, 41, 721-724. doi:10.1016/S0040-4039(99)02125-5

[17] Pon, R.T., Yu, S. and Sanghvi, Y.S. (1999) Multiple oligonucleotide synthesis in tandem on solidphase supports for small and large scale synthesis. Bioconjugate Chemistry, 10, 1051-1057. doi:10.1021/bc990063a

[18] Bofill, J.M. and Albericio, F. (1999) Understanding the structure/reactivity of aminium/uronium salts as coupling reagents in peptide synthesis. Tetrahedron Letters, 40, 2641-2644. doi:10.1016/S0040-4039(99)00262-2

[19] Hermkens, P.H.H., Ottenheijm, H.C.J. and Rees, D.C. (1997) Solid-phase organic reactions II: A review of the literature November 95-November 96. Tetrahedron, 53, 5643-5678. doi:10.1016/S0040-4020(97)00279-2

[20] Booth, S., Hermkens, P.H.H., Ottenheijm, H.C.J. and 
Rees, D.C. (1998) Solid-phase organic reactions III: A review of the literature November 96-December 97. Tetrahedron, 54, 15385-15443. doi:10.1016/S0040-4020(98)00968-5

[21] Zaidi, J.H., Naeem, F., Khan, K.M., Iqbal, R.Z.-U. (2004) Synthesis of dithioacetals and oxathioacetals with chiral auxiliaries. Synthetic Communications, 34, 2641-2653. doi:10.1081/SCC-200025627

[22] Washington, J.A.H. and Sutter, V.L. (1980) Dilution susceptibility test: Agar and macro-broth dilution procedures.
In: Lennette, E.H., Balows, A., Hausler, W.J.Jr. and Truant J.P., Eds., Manual of Clinical Microbiology, 3rd Edition, American Society for Microbiology, Washington, DC, 453-458.

[23] Carron, E.A., Maran, J.M., Montero, L., Fernandozalgo, A. and Dominiguez, A. (1987) Antimicrobial properties of some extracts obtained from some Mediterranean plants of medicinal value. Plantes Médicinales et Phytothérapie, 21, 195-202. 